\section{Cyberscience: Die Zukunft der Wissenschaft im Zeitalter der Informations- und Kommunika- tionstechnologien}

\author{
von Michael Nentwich, Institut für Technik- \\ folgenabschätzung der Österreichischen \\ Akademie der Wissenschaften, Wien
}

\section{In einem breit angelegten Projekt widmet sich das Institut für Technikfolgenabschät- zung der Aufarbeitung jener Veränderungen, die sich in den Wissenschaften aufgrund des verstärkten Einsatzes von Informations- und Kommunikationstechnologien abzeich- nen.}

Unser Ausgangspunkt ist eine Erfahrung, die praktisch alle wissenschaftlich Tätigen während des letzten Jahrzehnts gemacht haben: Der Computer ist mittlerweile aus dem Arbeitsalltag kaum mehr wegzudenken. Auch wenn zwischen den verschiedenen Disziplinen bemerkenswerte Unterschiede bestehen, so scheint es doch kaum mehr WissenschaftlerInnen zu geben, die nicht in irgendeinem Zusammenhang mit Computern arbeiten. Selbst in den Sekretariaten jener immer kleiner werdenden Schar, die Computer selbst nicht benutzen, stehen heute durchwegs Geräte, die zumindest für Textverarbeitung eingesetzt werden und die Schreibmaschine mittlerweile fast vollständig abgelöst haben. Auf dem anderen Ende eines "Nutzungs"Kontinuums stünden jene, für die der PC auf dem Schreibtisch die tägliche Arbeit entscheidend bestimmt, die ihn also nicht nur als bequeme Schreibmaschine verwenden, sondern sein großes Potential in vielfacher Hinsicht ausnutzen. Eine entscheidende Rolle spielten vor allem die zunehmende Vernetzung der Computer, die Entwicklung des Internet und das in den letzten Jahren sich explosionsartig entwickelnde World Wide Web (WWW), welches einen beinahe intuitiven Zugang zur "virtuellen" Welt ermöglicht.

Textverarbeitung auf dem PC, elektronische Post (E-mail), elektronisches Publizieren und online-Datenbanken sind dabei nur einige Begriffe, die bereits eingeleitete Veränderungen des Kommunikationsverhaltens in der Wissenschaft bezeichnen. Doch das ist aller Voraus- sicht nach erst der bescheidene Anfang, denn schon jetzt kündigen sich einige weitere Entwicklungen an: virtuelle Workshops, onlineBegutachtung von Texten, "intelligente Suchagenten", global vernetzte Datenbanken, digitale Bibliotheken, "hypertextuelle Zettelkästen" usw. Noch befinden wir uns in einem teilweise experimentellen Stadium, aber es ist bereits abzusehen, daß sich einiges durchsetzen und nicht nur das Verlags- und Bibliothekswesen, sondern den Wissenschaftsbetrieb im allgemeinen und die wissenschaftliche Kommunikation im besonderen nachhaltig beinflussen wird. Nicht nur in den USA wurde für diese Entwicklung die einprägsame Bezeichnung "PostGutenberg Galaxis" geprägt und gemutmaßt, daß wir uns vor einer vierten kognitiven Revolution (nach der Erfindung der Sprache, der Schrift und des Buchdrucks) befinden.

Das Projekt setzt es sich zum Ziel, über die schon heute festzustellenden, inkrementellen Änderungen im Wissenschaftsbetrieb hinaus mögliche bzw. wahrscheinliche zukünftige Entwicklungen $\mathrm{zu}$ antizipieren und einzuschätzen. Koexistieren doch geradezu messianische, technik-euphorische Plädoyers auf der einen Seite und große Skepsis sowie sogar eine Tendenz zur Computer-Verweigerung bei manchen WissenschaftlerInnen auf der anderen Seite. Angesichts dessen ist eine kritische Evaluierung und vorsichtige Extrapolation bestehender Trends angebracht. Zwei aufeinander bezogene Thesen stehen im Zentrum der Arbeit:

- Der Übergang zur Cyberscience hat das Potential, in allen Dimensionen wissenschaftlicher Aktivität einschließlich des organisatorischen Rahmens Veränderungen hervorzubringen, und

- $\quad$ es wird sich dabei nicht ausschließlich um quantitative, sondern auch um qualitative Veränderungen in der Wissenschaft handeln.

Ein erstes systematisches Screening des Wissenschaftsbetriebs hat bereits deutlich gemacht, daß sowohl sein organisatorischer Rahmen wie auch die Wissensproduktion (Informationsbeschaffung, Datengewinnung, -verwaltung, Informationsverarbeitung, Ergebnisdarstellung) sowie die Formen der wissenschaftlichen Kommunikation (Kooperation, Diskursformen, Veröffentlichungswesen) und schließlich auch 
die Wissensvermittlung (Lehre) von den hier unter dem Stichwort Cyberscience zusammengefaßten Entwicklungen direkt betroffen sind.

Im Laufe des Projekts werden nun eingehend einige Bereiche des Wissenschaftsbetriebs herausgenommen und einer intensiven Untersuchung ausgesetzt, wobei folgende Fragen im Zentrum stehen:

- Sind die beobachteten Veränderung qualitativer Natur? Mit anderen Worten: Betreffen sie nicht nur quantitative Aspekte wie etwa die Publikationsgeschwindigkeit und -menge? Qualitative im hier gemeinten Sinn wären etwa strukturelle Wandlungen im organisatorischen Umfeld, in dem Wissenschaft betrieben wird, ebenso wie grundlegende Veränderungen in Hinblick auf die Rollen, die von den verschiedenen Akteuren des Wissenschaftssystems eingenommen werden, oder jene, die die Spezifika der wissenschaftlichen Kommunikation bzw. des Prozessierens wissenschaftlicher Erkenntnisse (Arbeitsweisen) betreffen. Eine fundamentale qualitative Veränderung wären schließlich Verschiebungen in den Inhalten dessen, was überhaupt erforscht wird.

- Haben die neuen Anwendungen das Potential, die eher traditionellen Formen zu ersetzen oder lediglich zu ergänzen? Welche Faktoren beeinflussen diesen Prozeß?

- Wie können wir die offenkundigen zeitlichen, disziplinären und örtlichen Unterschiede erklären?

- Welche Konsequenzen sind daraus für die Wissenschafts- und Forschungspolitik zu ziehen?

Diesen Leitfragen wird unter anderem in folgenden Untersuchungsfeldern nachgegangen, wobei wir uns eines methodischen Mix aus Aufarbeitung unterschiedlichster Literaturstränge (Wissenschaftssoziologie, Bibliothekswissenschaften, Interökonomie, Kommunikationswissenschaft etc.) und empirischen Erhebungen (Interviewserien, technische Marktanalyse, Expertenworkshop) bedienen:

(1) Die Konsequenzen der CyberscienceAnwendungen auf die räumliche Verteilung und infrastrukturelle Ausstattung von Wissenschaft. Stichworte in diesem Bereich sind beispielsweise virtuelle Institute und Labors (sog. "collaboratories"), virtuelle Bibliotheken und E-Konferenzen.

(2) Weiters sind nachhaltige Einflüsse auf die zukünftige Rollenverteilung im Wissenschaftsbetrieb zu erwarten. Insbesondere die Rolle der wissenschaftlichen Bibliotheken ist in tiefgreifendem Wandel begriffen (vom "librarian" zum "cybrarian"), ebenso wie die der Verlage, aber auch die Anforderungen an die einzelnen WissenschaftlerInnen verändern sich und könnten eventuell zur Ausdifferenzierung neuer Rollen, wie der des "scientific information broker", führen.

(3) Aufgrund der fortschreitenden Elektronisierung der Textproduktion stellen sich spannende Fragen im Zusammenhang mit der Wissensrepräsentation. Liegt die Zukunft statt auf Papier im elektronischen Raum, statt in linearen Texten in "Hypertexten" und in verteilten Wissensdatenbanken?

(4) Womit bereits das nächste Thema angerissen ist: die Zukunft des wissenschaftlichen Veröffentlichungswesens. Das Internet bietet interessante neue Möglichkeiten, die parallel zu bzw. sogar noch vor den Verlagen auch von nicht-kommerziellen Akteuren entdeckt wurden und es stellt sich die Frage, wie der sich abzeichnende Kampf um die Leserschaft ausgehen und damit, wie sich dieser Eckpfeiler des Wissenschaftsbetriebs weiterentwickeln wird.

(5) Eng damit verknüpft ist die Zukunft der Qualitätskontrollsysteme im Zeitalter der Cyberscience zu diskutieren, da nicht nur traditionelle Formen umgangen werden können (Stichwort: Preprint-Server), sondern auch neue Formen möglich gemacht wurden (Stichwort: offene Begutachtungssysteme).

(6) Der Einzug von Multimedia und Internet scheint weiters das Arbeitsumfeld der WissenschaftlerInnen als Lehrpersonal entscheidend $\mathrm{zu}$ verändern. Wir gehen der Frage nach, inwieweit die "virtuelle Universität" auf die Wissenschaft rückwirkt.

Näheres zum Projekt findet sich auf der unten angegebenen Homepage des Instituts sowie in 
Form eines ausführlichen Arbeitspapiers, das kürzlich als Working Paper des Max-PlanckInstituts für Gesellschaftsforschung in Köln mit der Nummer 99/6 unter folgendem URL veröffentlicht wurde:

http://www.mpi-fg-koeln.mpg.de/publikation/ working_papers/wp99-6/.

\section{Kontakt}

Dr. Michael Nentwich

Institut für Technikfolgenabschätzung

Österr. Akademie der Wissenschaften

Strohgasse 45/5, A-1030 Wien

Tel. + 43151581 6583; Fax. + 4317108983

E-mail: mnent@oeaw.ac.at

Internet: http://www.oeaw.ac.at/ita

》

\section{POST-Studie über die Möglich- keiten des Einsatzes von luK- Technologien in Regierung und Verwaltung: "Electronic Go- vernment"}

\author{
von Ingrid von Berg, ITAS
}

Das Parliamentary Office of Science and Technology (POST), London, hat einen Bericht zu einer in den Jahren 1998/1999 durchgeführten Studie zum Einsatz der Informations- und Kommunikationstechnologien in Regierung und Verwaltung "Electronic Government - Information Technologies and the Citizen" veröffentlicht. IuKTechnologien hätten das Wirtschaftsleben revolutioniert und würden zu einem Wandel des Verhältnisses zwischen Unternehmen und Kunden führen. Im öffentlichen Sektor seien die Auswirkungen bisher weniger dramatisch, weshalb sich die Frage stellt, ob die Möglichkeiten der IuK-Technologien im Bereich der öffentlichen Dienstleistungen gegenwärtig ausgeschöpft werden.

Der wesentliche Anstoß für eine politische Initiative der britischen Regierung für die electronische Bereitstellung von öffentlichen Dienstleistungen ging von dem "government.direct"- Programm des Central IT Unit
(CITU) des Cabinet Office aus. Das Programm wurde im November 1996 durch ein Green Paper initiiert und mit einem interaktiven online-Diskussionsprozeß mit den Bürgern fortgesetzt (wobei dafür Sorge getragen wurde, daß auch Bürger, die keinen Zugang zu PC und Internet haben, sich beteiligen konnten). Als Folge dieses Prozesses wurde eine Reihe von Pilot-Projekten in Angriff genommen, um die zugrundeliegenden Technologien sowie die Reaktionen der Öffentlichkeit und der Wirtschaft zu testen und zum Experimentieren mit neuen Anwendungen der ICT im öffentlichen Bereich anzuregen.

Die Blair-Regierung führt diese Initiative fort, paßt das Konzept jedoch in einen sehr viel breiteren Rahmen ein im Sinne eines "better government" und parlamentarischer und verfassungsmäßiger Reformen. Konkrete Schritte für einige dieser Inititativen sind bereits in die Wege geleitet worden, z.B. für das schottische Parlament und die walisische Nationalversammlung Der Bericht geht im Detail auf die hier angesprochenen Entwicklungen ein. Premierminister Blair hat als Ziel gesetzt, daß im Jahre 2002 25\% aller Transaktionen der Regierung und der öffentlichen Verwaltung elektronisch erfolgen sollen.

Ausgehend von der Darstellung der gegenwärtig realisierten Anwendungen der Informations- und Kommunikationstechnologien in Regierung und öffentlicher Verwaltung wird im Bericht dann die Frage aufgeworfen, ob der Einsatz dieser neuen Technologien anders organisiert würde, wenn sie heute "von Grund auf neu" entworfen werden würden - im Gegensatz zum bestehenden System, das auf Strukturen und Funktionen basiert, die sich herausgebildet haben, bevor man überhaupt an Computer gedacht hat.

Wenn die Erfahrungen aus der Wirtschaft zutreffen, dann, so folgert der Bericht, bieten IuK-Technologien die Chance, die Regierungsund Verwaltungsaktivitäten nach völlig neuen Gesichtspunkten umzugestalten (re-engineering), d.h. neue Management-Modelle einzuführen. Es wird eine Reihe von Vorschlägen diskutiert - so könnten die Systeme zum Beispiel in Hinblick auf Prozeßabläufe, die Lebensabschnitte (life events) eines Bürgers (Geburt, Schule, Arbeitsplatz etc.) oder nach dem Gesichtspunkt einer Dienstleistungskette für den 